\title{
Space Charge and Dipoles in Polyvinylidenefluoride*
}

\author{
W. Eisenmenger, H. Schmidt and B. Dehlen \\ Universität Stuttgart \\ 1. Physikalisches Institut, Pfaffenwaldring 57, \\ D-70550 Stuttgart, Germany
}

Received 2 April, 1998; Received in revised form 30 November, 1998.

\begin{abstract}
The properties of space charge (homocharge) and dipoles (heterocharge) in various electrets have been in the focus of interest since the investigations of B. Gross on carnauba wax. In this article we present a review on the role and the interaction of charges and dipoles in the polymeric electret polyvinylidenefluoride (PVDF). It covers the importance of charge injection for the orientation of the dipoles at low fields as well as the role of charge trapping for the stabilization of the polarization. Investigations of the thermal stability of the polarization and the charge balance are leading to a detailed analysis of the depth and localization of the charge traps. Furthermore, it was possible to identify the space charge as ions created electrochemically during the poling process.
\end{abstract}

\section{Introduction}

Pioneering work in electret research in the years between 1940 and 1960 especially by Bernhard Gross [1,2] has been comprehensively summarized in his review articles [3,4]. These investigations, predominantly carried out with carnauba wax, led to the confirmation and better knowledge of the so called "two charge model" of electrets. This model explains the observation that the polarization charges at the surfaces of an electret can either be of the same or of the opposite polarity as the previously applied electric field by the existence of two independent types of charge, the so called homocharge and heterocharge respectively. While the homocharge could be assigned to real charges injected from the sample surfaces, the heterocharge which fills the whole bulk of the samples could be identified with the formation or orientation of dipoles or the extraction of opposite excess charge $[2,5,6,7]$.

After the discovery of the strong piezoelectric effect induced by applying high electric fields to the polymer electret polyvinylidenefluoride (PVDF) [8] a long lasting controversy on the role of charges and dipoles in this material began [9]. Because many publications are available on the basic properties $[10,11]$ and the various applications $[11,12]$ of PVDF we will concentrate on the facts which are important for the understanding of the underlying physical processes.

PVDF is a semicrystalline polymer consisting of linear chains with the sequence $\left(\mathrm{CH}_{2}-\mathrm{CF}_{2}\right)_{\mathrm{n}}$ as shown in Fig. 1. By stretching the polymer the approx. $50 \%$ crystalline phase can be converted predominantly from the thermodynamically stable $\alpha$-phase into the $\beta$-phase in which the polymer chains exhibit the zigzag-conformation shown in Fig.1 and are ordered in a quasi-hexagonal lattice. The electronegativity difference of the fluorine and hydrogene substituents located at opposite sides of the polymer chain results in a large dipole moment perpendicular to the chain direction. In a virgin PVDF sample these dipoles are aligned in randomly oriented domains. After the application of a high electric field in the order of $100 \mathrm{MV} / \mathrm{m}$ to a PVDF film which is also denominated as poling, the sample exhibits piezoelectric properties. This can be attributed to the permanent orientation of the dipoles in the crystalline $\beta$-phase as indicated by infrared spectroscopy [13] and x-ray [14] measurements. The importance of the dipole orientation for the formation of polarization

*Dedicated to Prof. Bernhard Gross 
and piezoelectricity in PVDF was recognized immediately [15]. On the other hand, the role of the charges in this context was more difficult to elucidate, although the necessity of charge injection was also realized early [16]. Materials similar to PVDF, but with faster polarization dynamics and therefore better suited for certain measurements are the copolymers of vinylidenefluoride and trifluorethylene $\mathrm{P}$ (VDF-TrFE) which were also used in some of our experiments. The following report concentrates on the results mainly obtained in our institute during the last 15 years.

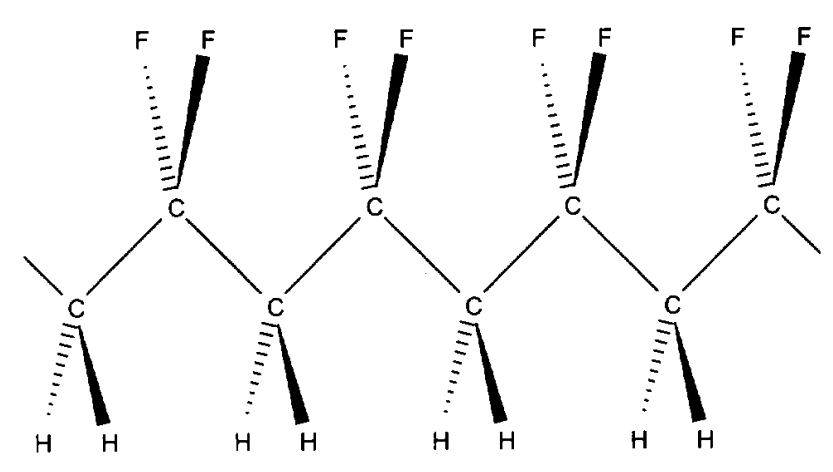

Figure 1. Molecular structure of a PVDF polymer chain.

\section{Experimental}

The key for the understanding of the charge and polarization properties in polymer films is the measurement of the their spatial distribution after and preferentially also during the poling process. For this purpose, different techniques were developed around 1980 which are reviewed in [17]. Here we describe the so called PPS (piezoelectically generated pressure step wave) method [18]. As shown in Fig.2 a pressure step generated by a quartz crystal propagates into the polymer film. The resulting signal detected with a wide band oscilloscope is directly proportional to the polarization plus the integral over the charge density. By a modification of the setup shown in Fig.2 it is even possible to study the evolution of the polarization during the poling process, i.e. in-situ. Here the PVDF sample is covered with a thin PET film which carries the poling electrode.

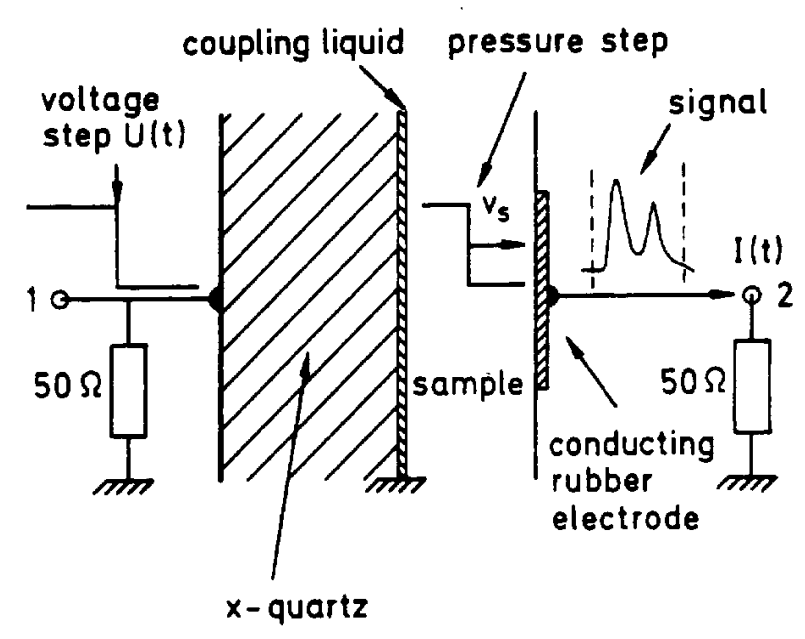

Figure 2. A schematic sketch of the PPS setup [18].

\section{Results and Discussion}

\section{III.1 Charge injection during poling}

Whereas in charging carnauba wax heterocharge dominates homocharge [5], the poling process in PVDF in general is accompanied by charge injection.

Charge injection is particularly strong during poling at field strengths below approx. $100 \mathrm{MV} / \mathrm{m}$ as was measured by the in-situ-PPS method [19]. Fig. 3a shows the polarization development during the poling of a 50 $\mu \mathrm{m}$ thick $\beta$-PVDF film at $40 \mathrm{MV} / \mathrm{m}$. Immediately after field application a rectangular signal over the full sample thickness is observed indicating a homogeneous dipole orientation and compensation charges located at the sample surfaces. After a few minutes the shape of the polarization profile rounds due to charge injection. After 30 min the charges are accumulating left and right at the boundaries of a central polarization zone. This distribution becomes stable after 75 min, i.e. it remains unchanged even after longer poling times. This indicates the injected charges do not traverse the entire sample but are trapped at the boundaries of the polarized region. At a field strength of $80 \mathrm{MV} / \mathrm{m}$, see Fig. $3 \mathrm{~b}$, almost the same behaviour is found but the polarized region extends more close to the film surface still with a polarization free boundary zone left. In this case the charges are trapped earlier after injection. After 
zero voltage at the sample, see Fig. 4, the dipole orientation relaxes resulting in the release of homocharges as excess charge between sample surface and polarization zone which slowly decays within some $100 \mathrm{~s}$. For comparison, the PPS signal during poling polyvinylchloride

a)

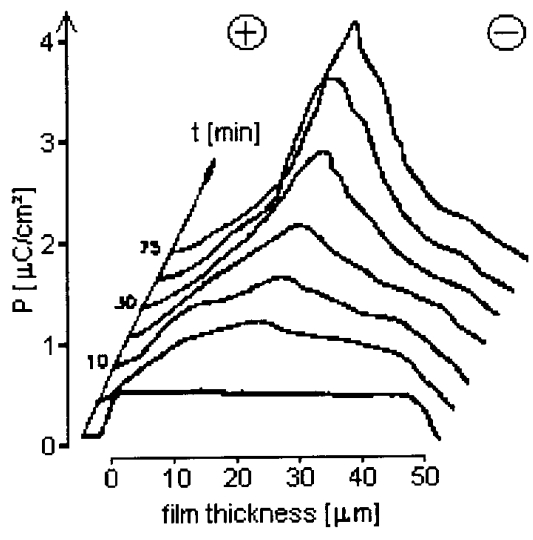

(PVC) is shown in Fig. $5[7]$. In this case no charge injection takes place, but after approx. 3 hours charge extraction from the bulk towards the surfaces can be observed in the form of heterocharge as indicated by the increasing PPS signal near the sample boundaries.

b)

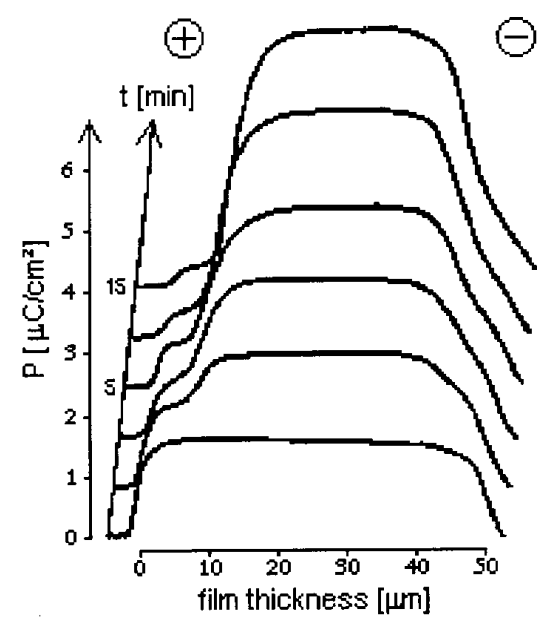

Figure 3. Development of inhomogeneous polarization profiles during poling of PVDF with low field strengths [19]: a) E = $40 \mathrm{MV} / \mathrm{m}$; b) $\mathrm{E}=80 \mathrm{MV} / \mathrm{m}$.

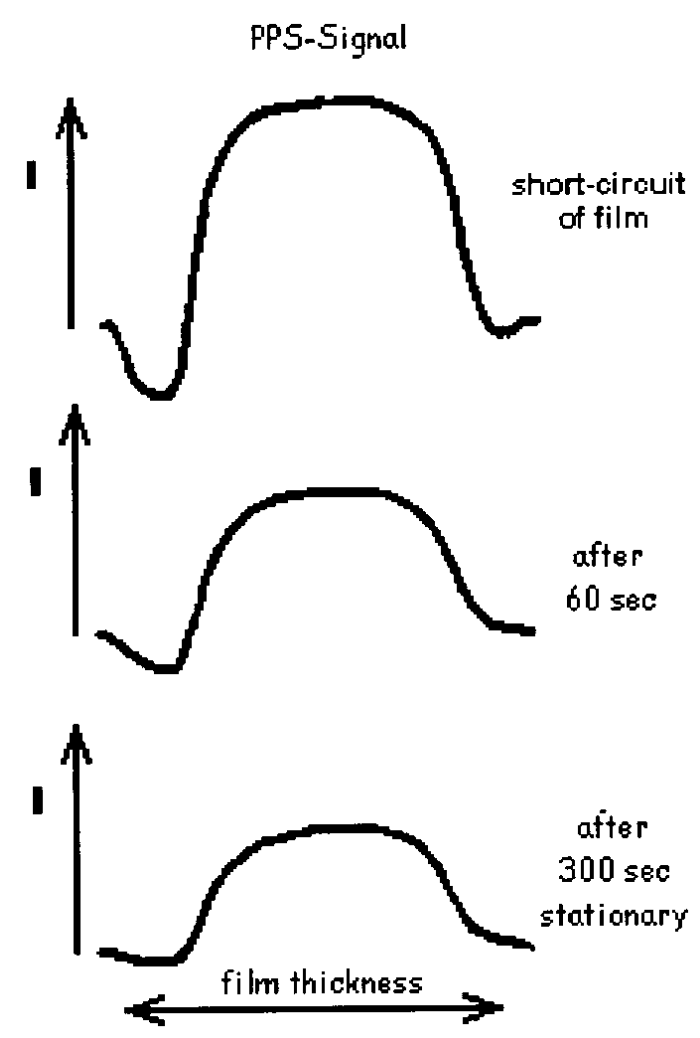

Figure 4. Development of PPS signal after short-circuit of the electrodes: excess charge exists near the inner sample boundaries [21].

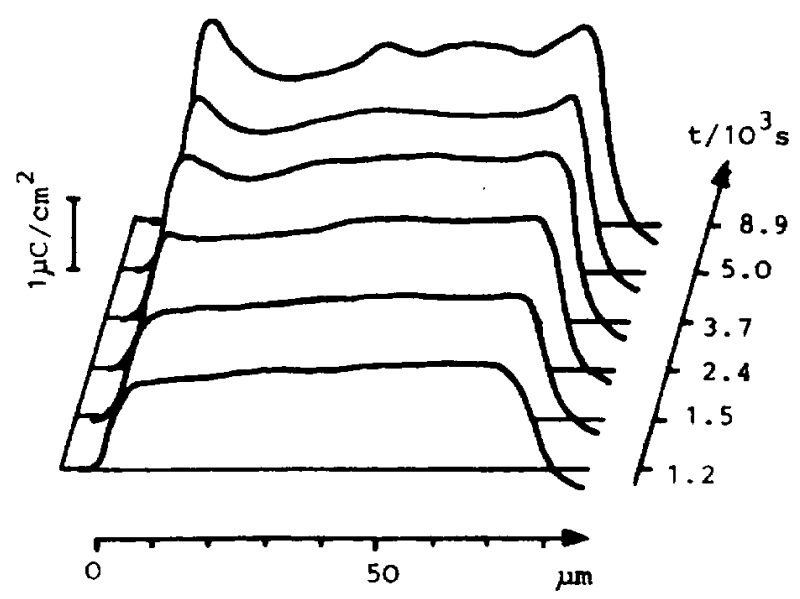

Figure 5. Polarization development during the application of an electric field of $50 \mathrm{MV} / \mathrm{m}$ to an $80(\mu \mathrm{m})$ thick PVC film at $75^{\circ} \mathrm{C}$ [7]. After approx. 1h the polarization near the boundaries of the sample increases indicating charge extraction from the bulk of the PVC.

Injected charges are also important for the polarization behaviour during poling of prepolarized PVDF films with fields in opposite direction [20,21]. Fig. 6 shows the polarization distribution observed in an 38 $\mu \mathrm{m}$ thick PVDF film prepoled $330 \mathrm{~s}$ at $180 \mathrm{MV} / \mathrm{m}$ dur- 
ing poling with $-60 \mathrm{MV} / \mathrm{m}$ for $20000 \mathrm{~s}$ [21]. While the polarization near the film boundaries does not change its direction, polarization inversion is observed in the center of the sample. It is important to notice that this inversion is possible even though the reverse field is well below the coercive field and the prepoling field. Without charge injection and charge trapping at the polarization zone boundaries a homogenous polarization decay should have been expected.
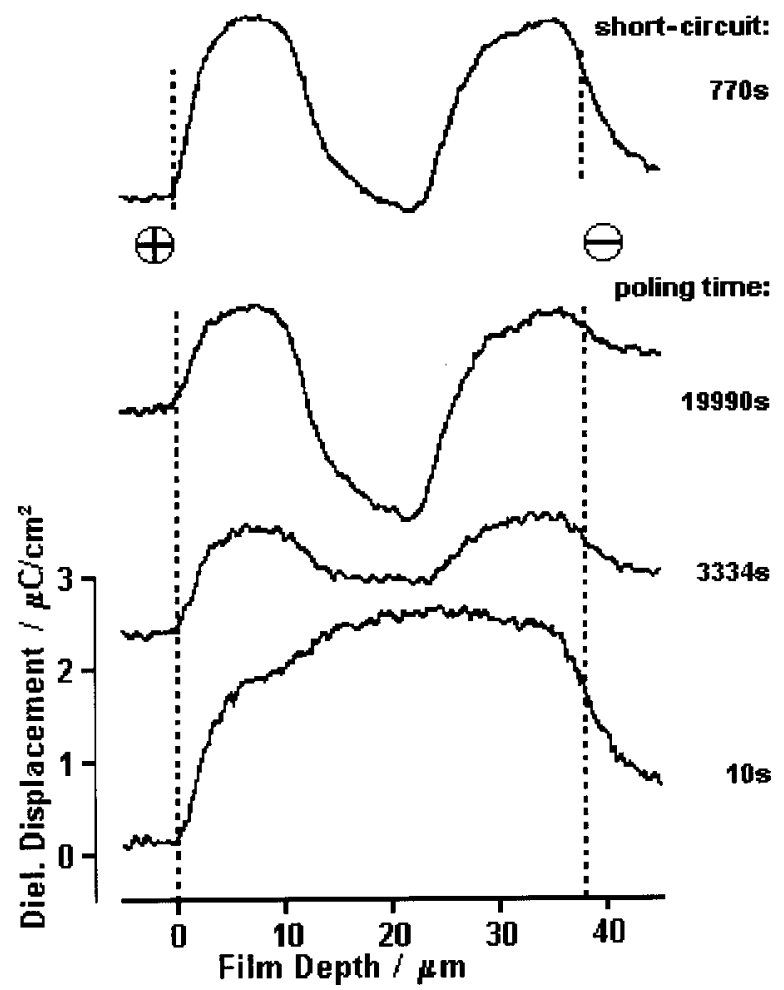

Figure 6. Formation a trimorph structure after poling prepolarized PVDF-films with an opposite field [21].

Not only the polarization development during poling, but also the polarization stability is affected strongly by the injected charges [22]. Fig. 7 compares the dielectric displacement during poling and the remanent polarization measured afterwards of two differently contacted $\mathrm{P}(\mathrm{VDF}-\mathrm{TrFE})$ samples at identical field strengths. One sample was directly contacted with evaporated metal electrodes, at the other sample the field was applied via blocking electrodes made of polyethylenetherephtalate (PET). Since PET is a very good insulator, charge injection in the $\mathrm{P}(\mathrm{VDF}-\mathrm{TrFE})$ was prevented in the latter case. As shown in Fig. 7a the dielectric displacement during poling is the same for both samples indicating equal field strengths. But after removing the external field the polarization in the sample with blocking electrodes decays much more rapidly resulting in nearly zero remanent polarization (Fig.7b). This shows that injected charges are somehow needed for the stabilization of the polarization in PVDF and its copolymers.
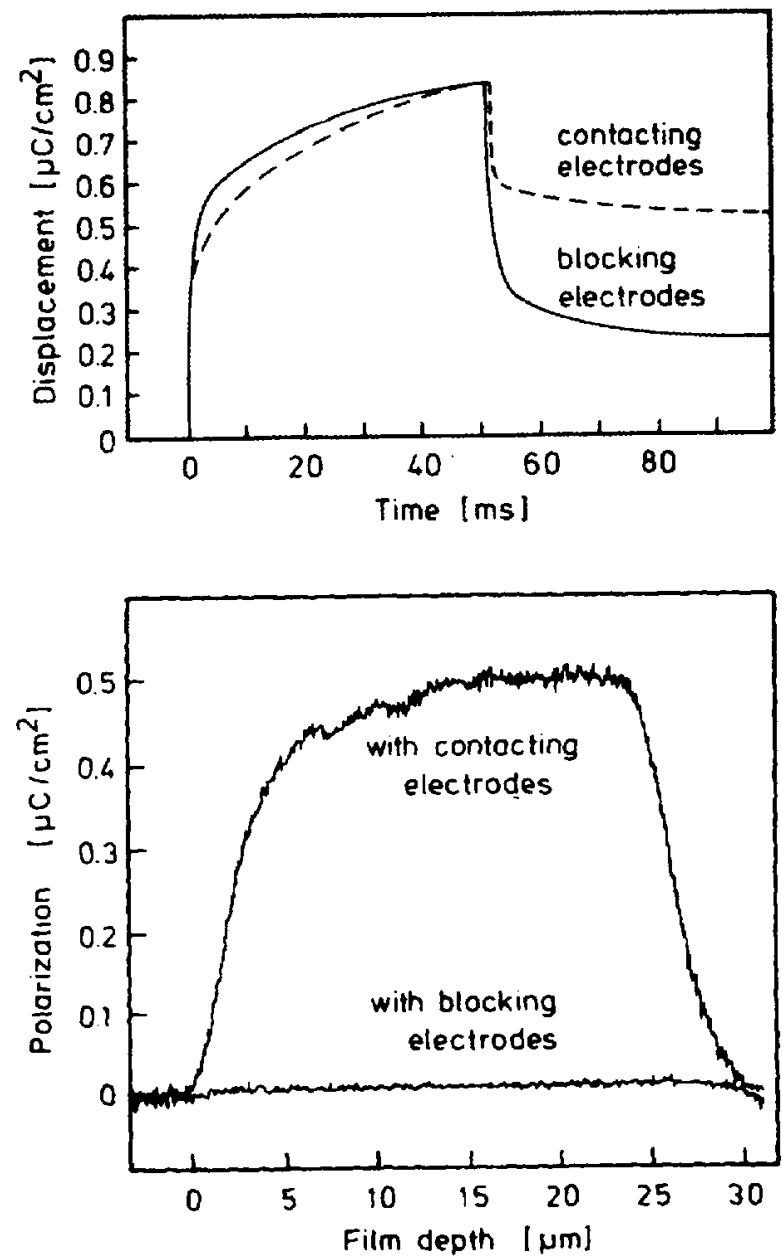

Figure 7. Top: Dielectric displacement measured during poling with and without blocking electrodes. Bottom: Polarization distributions after poling with and without blocking electrode [22].

\section{2 Dipole (and charge) dynamics}

Additional information about the charge-dipole interaction can be revealed if the polarization and depolarization dynamics are studied. Similar to the experiments of Gross in carnauba wax $[1,5]$ where the heterocharge showed faster decay than the homocharge, our measurements show that the dipoles are reaching their equilibrum faster than the charges in PVDF.

As described above, the charges injected during the poling process must be trapped somehow at the boundaries 
of the polarized region and thus play an important role for the polarization stabilization. In this case a time delay between immediate dipole orientation and polarization stabilization can be expected because of the limited charge injection at the electrodes. In contrast to this a polarization stabilization by a pure dipole-dipole interaction should result in immediate correspondence of momentary and permanent polarization. But in fact a significant time delay has been observed in short time polarization experiments with unpolarized and prepolarized PVDF [23] and P(VDF-TrFE) [24] film material. In the prepolarized material polarization reversal has been studied. As an example, Fig. 8 shows the results obtained by poling unpolarized P(VDF-TrFE) films at different field strengths. While at $10^{-5} \mathrm{~s}$ a clearly significant dipole orientation is achieved at least at the higher fields, no permanent polarization can be observed. After longer poling times, permanent polarization remains after switching off the field, but is always delayed with respect to the dielectric displacement by a time factor of 2 to 3. A similar behaviour has been observed in switching experiments [24] and also for pure PVDF [23].
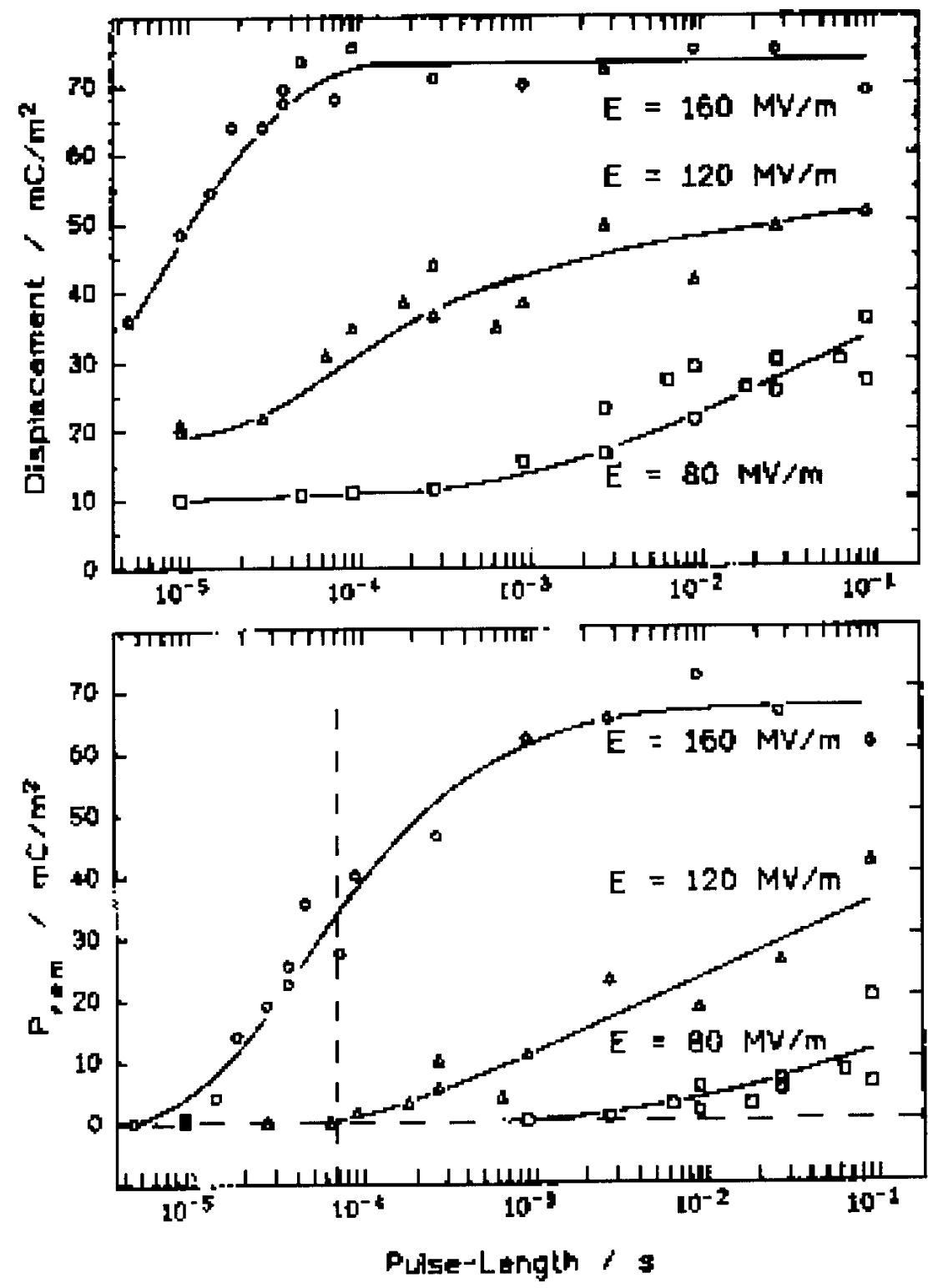

Figure 8. Time development of the displacement (top) and the remanent polarization (bottom) dependent on the applied HV pulse length. Previously unpoled films of P(VDF-TrFE) 75/25 copolymer poled at different field strengths [23]. 
Charge-dipole interaction affects not only the temporal polarization behaviour during poling but also during thermal depolarization with short heat pulses [25]. By some improvements of the PPS apparatus it was possible to apply heat pulses with a temperature difference of about $20^{\circ} \mathrm{C}$ within approx. $10 \mathrm{~ms}$ to our samples and to measure the resulting polarization with a repetition rate of approx. $400 \mathrm{~s}^{-1}$. Fig. 9 shows the polarization response to such a short heat pulse. The polarization follows the temperature without delay as to be expected for a pyroelectric material, but with a much higher amplitude as could be calculated from the pyroelectric coefficient of PVDF. This anomalous behaviour is called the short-time-pyroelectricity of PVDF. It can be understood if during such a heat pulse the dipoles loose their orientation but the charges trapped at the boundaries of the previously polarized crystallites are not able to leave their positions in the short time available. Then the dipoles can be realigned in the field of the remaining charges. Realignment of dipoles in the field of trapped charges is also the reason for the anomalous recovery of polarization after cooling down PVDF films which had been thermally depolarized near the melting point [26].

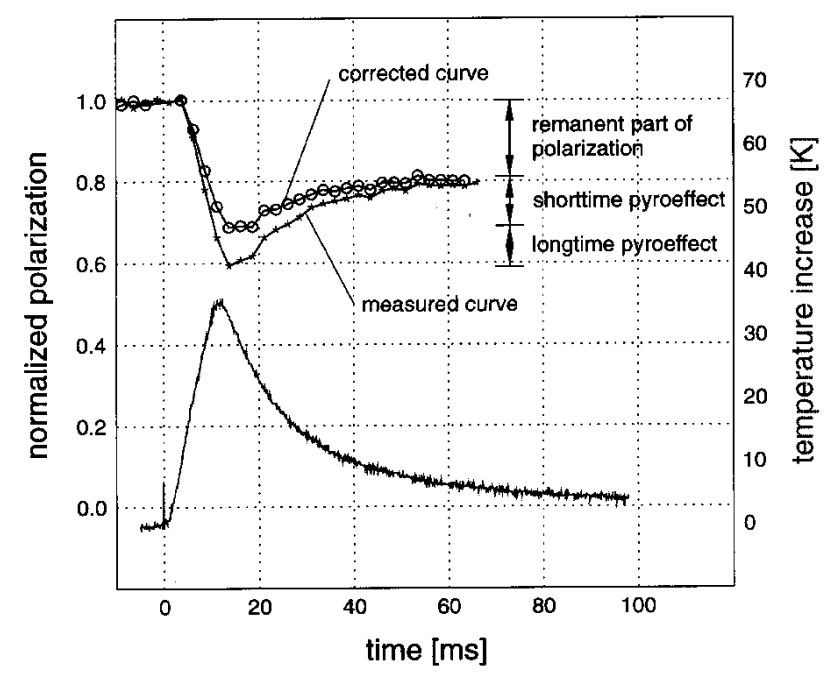

Figure 9. PPS Signal during a short heat-pulse [25] showing the short time pyroelectric effect.

\section{III.3 The charge trapping model}

It is obvious that for the stabilization of a permanent and macroscopic dipole orientation in PVDF a spe- cific mechanism is required as for hard ferromagnetism. In the case of magnets the dipole-dipole interaction plays an important role along with a strong crystal field anisotropy, domain wall pinning and a high activation energy for thermal domain wall creation. In the case of electrets an additional stabilization mechanism is possible by trapping of real charges at the surfaces of the polarized crystallites. These charges not only compensate the dipoles reducing their external stray field but also charges and oriented dipoles are mutually attracted by electrostatic forces.

Our measurements described above show the necessity of charge injection for polarization stabilization and the alignment of the injected charges at the polarized region. This leads to the conclusion [18], that the polarization stability in PVDF is mediated by the trapping of injected charges in coulomb traps at the boundaries of the polarized crystallites ${ }^{1}$. This charge trapping is illustrated in Fig. 10 for the two possible cases of pure polarization compensation and complete neutralization. For pure compensation, trapped charges are located at the crystallites near the sample boundaries requiring significantly less charges than for a complete neutralization where each individual crystallite even in the bulk of the sample is locally compensated.
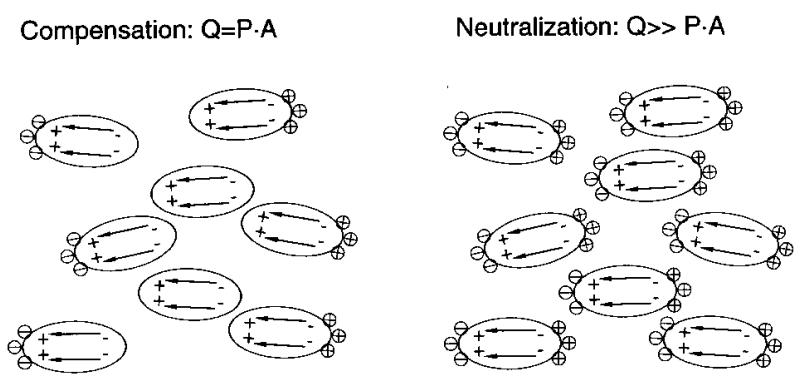

Figure 10. Illustration of polarization stabilization in the charge trapping model. Left side: pure compensation. Right side: neutralization.

We were also able to simulate the polarization stabilization by charge trapping with a simple mechanomagnetic model as shown in Fig. 11 [27]. As expected for PVDF the pure dipole-dipole interaction is not sufficient for the stabilization of a homogeneous dipole orientation (top subfigure) whereas "trapped charges at

\footnotetext{
${ }^{1}$ For ions as charge carriers as implied by some later discussed experiments, not only electrostatic attration but also an at least partly chemical bonding is possible.
} 
crystallite surfaces" stabilize the polarisation (bottom subfigure).
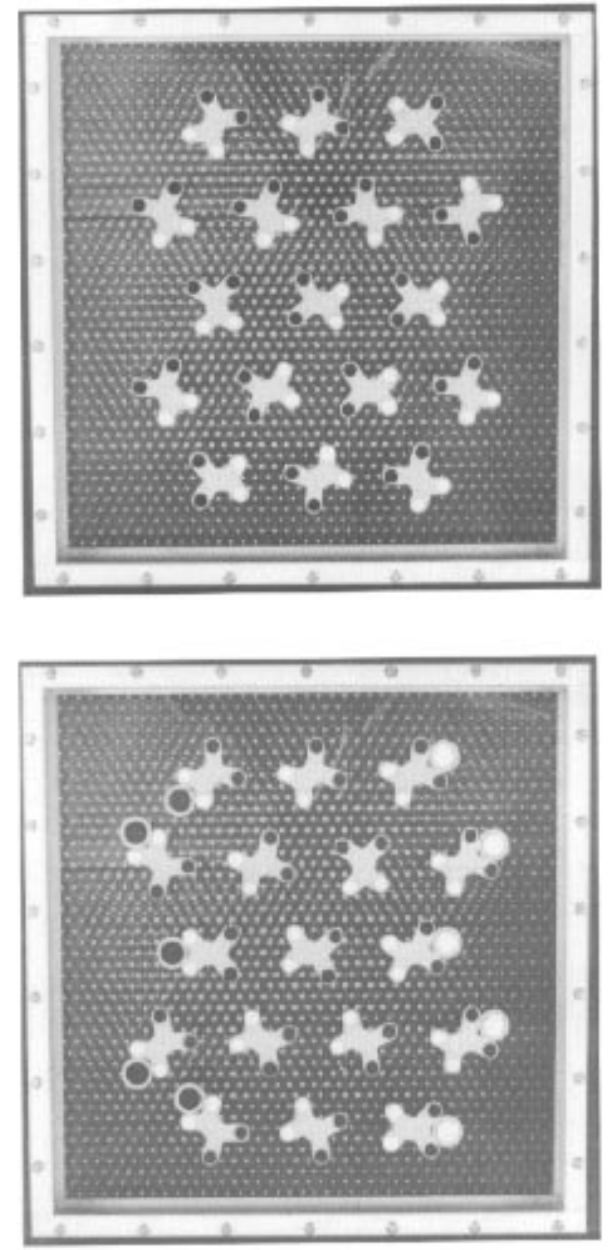

Figure 11. Tllustration of polarization stabilization by charge trapping with a magneto-mechanical model. The charges and dipoles are simulated by small magnets appropiately mounted on plastic floating on an air cushion. The "dipoles" are formed resembling the PVDF chain dipoles and can rotate around a fixed center whereas the "charges" can move freely. Top: Without "charges" the "dipoles" are randomly oriented. Bottom: With "charges" present at the boundaries the "dipoles" are all aligned along one direction [6].

\section{III.4 Depth and localization of the charge traps}

Although it is quite clear from the above stated results, that injected charges trapped at the boundaries of the polarized crystallites play a crucial role for the polarization stabilization in PVDF, the microscopic nature of the charge traps is yet beyond our knowledge. Two steps towards this goal are the investigation of the depth of the traps, i.e. the binding energies of the charges and of their spatial distribution depending on the poling conditions [28].

Binding energies can be examined by thermally stimulated discharge or depolarization measurements [11]. For the analysis of the broad energy distributions present in PVDF it was however necessary to develop a special mathematical technique [29]. The result of thermal depolarization measurements evaluated this way shows Fig. 12 [28]. It is clearly visible, that PVDF films poled at low field strengths exhibit one maximum in the energy distribution around $1,7 \mathrm{eV}$ whereas in films poled at high fields the typical binding energy is $2,3 \mathrm{eV}$. Samples poled at intermediate fields show a mixture of both cases. These binding energy values agree well with the observed high long-time-stability of the polarization.

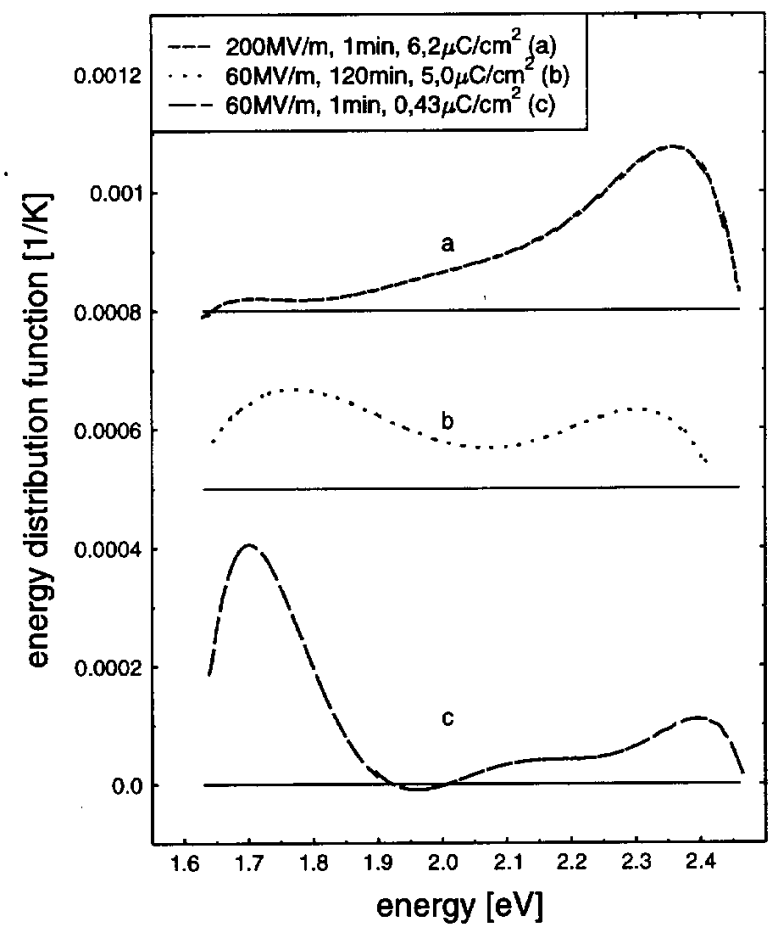

Figure 12. Binding energies of the charge traps in PVDF films poled at different field strengths [28].

A distinction between the global compensation and the local neutralization of the polarization is possible by evaluating the charge flow during poling. For a pure compensation, the charge density equals the polarization, i.e. $\mathrm{Q}=\mathrm{P} \cdot \mathrm{A}$ with $\mathrm{Q}=$ integral of the charging current, $\mathrm{P}=$ net polarization and $\mathrm{A}=$ sample area. In 
contrast to this, for complete neutralization, i.e. compensation of each individual crystallite, the amount of necessary charge is much higher. Fig. 13 [28] shows that for PVDF poled at high fields the condition for pure compensation $\mathrm{Q}=\mathrm{P} \cdot \mathrm{A}$ is almost fulfilled, whereas at lower fields significantly more charge is injected during poling implying at least partial neutralization.

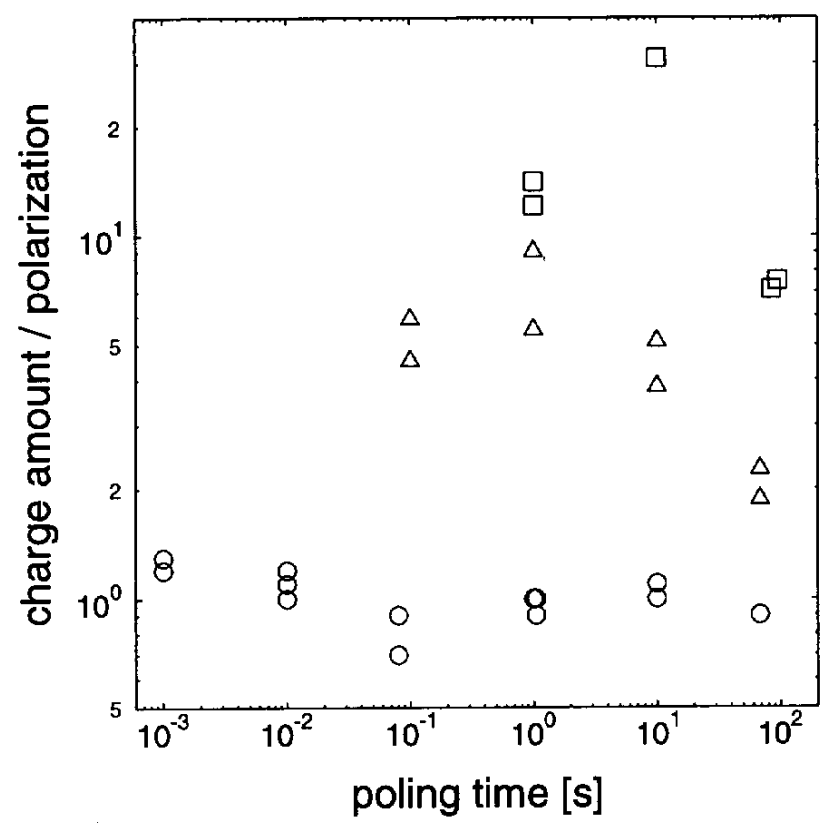

Figure 13. Neutralization or compensation? Charge balance for PVDF films poled at different field strenghts $(0$ : $\mathrm{E}=180 \mathrm{MV} / \mathrm{m}, \triangle \mathrm{E}=100 \mathrm{MV} / \mathrm{m}$ and $\amalg: \mathrm{E}=80 \mathrm{MV} / \mathrm{m})[28]$.

These measurements show that the poling conditions have strong influence on the formation of the charge traps. At high fields the dipole orientation is completed before a significant amount of charge is injected which results in trapping near the sample surface with high binding energies. In this case the dipoles in the center of the sample are not locally neutralized by trapped charges and should therefore be easier to depolarize. This indeed has been observed in different experiments. For example, after reverse poling a prepolarized PVDF film, see Fig. 6, the polarization is inverted in the sample center. Also if a prepolarized PVDF film is immersed in a polar solvent like acetone, the polarization decreases stronger in the sample center [30]. At low poling field strenghts a local field enhancement by charge penetration into the bulk of the material is necessary in order to reach the coercive field needed for dipole orientation. This results in strong charge injection and in trapping at lower binding energies at possibly less complete oriented dipoles.

\section{Ionic nature of the injected charges}

Another question is whether the charges in PVDF are electrons or ions, and if the latter, which type of ions. Generally, both types of carriers can be found in polymers [31]. The decrease of electrical conductivity with increasing hydrostatic pressure $[31,32]$ indicates that in PVDF ionic conduction is present. Ions can also be stronger bound in coulomb traps than electrons. The blocking experiments show that the ionic charges have to be created at the electrodes during the application of the poling field. A final test for ion generation and conduction should however also yield information about the polarity and type of ions as well as on the trapping process.

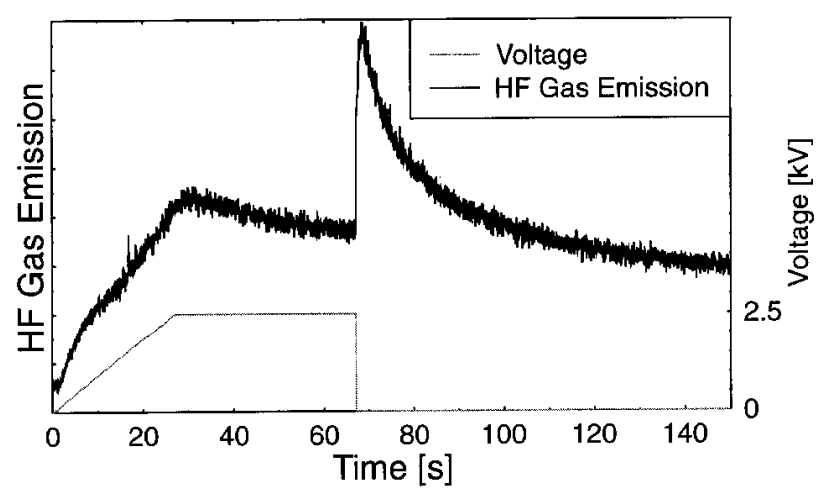

Figure 14. Hydrogen fluoride (HF) gas emission during poling a $25 \mu \mathrm{m}$ thick PVDF film covered with a porous gold electrode with a voltage of up to $2,5 \mathrm{kV}[38,37]$. The voltage is increased first linearly up to its maximum value, then held constant and finally switched to zero. In the first two stages the gas emission follows the poling voltage. Additionally, immediately after shortening more gas is emitted during the so-called "zero field emission".

If ions are created during poling, the formation of other, possibly gaseous, products in secondary reactions has to be expected. It was verified by poling PVDF with a porous, chemically inert gold electrode and measuring the amount and type of emitted gas with a mass spectrometer [33, 28], that during poling of PVDF hydrogen fluoride $(\mathrm{HF})$ and other gas molecules are created. As shown in Fig. 14 for HF, the gas emission increases with increasing poling voltage and saturates if the voltage is held constant as to be expected if the ions are generated electrochemically during poling. Besides that, immediately after switching to zero voltage 
an additional amount of gas is liberated. The origin of this so called zero-field-emission can be elucidated from the comparison of its time development with the polarization distributions measured after poling as shown in Fig. 15. The larger the distance between the polarization maximum and the permeable electrode through which the gas leaves the sample is, the more delayed is the peak of the zero-field emission. Therefore the source of the zero-field emission must be the polarized region and it can be concluded that it results from the detrapping of ions from shallow traps and subsequent gas formation and diffusion.

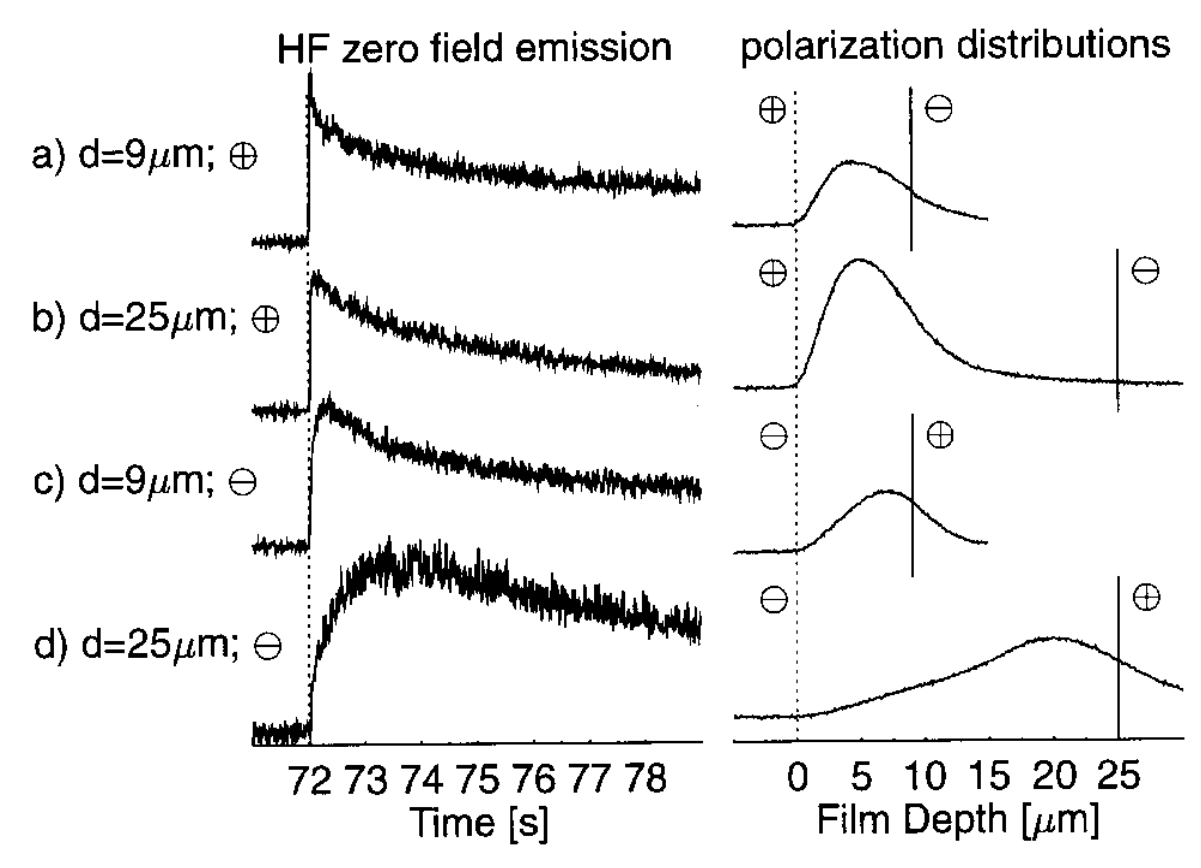

Figure 15. Left side: The HF zero field emission depending on film thickness and polarity at $\mathrm{T}=110^{\circ} \mathrm{C}[34]$. The poling field of $100 \mathrm{MV} / \mathrm{m}$ was shortened at $\mathrm{t}=72 \mathrm{~s}$ (dotted line) within $40 \mathrm{~ms}$. Right side: Mean polarization distributions measured after poling. The permeable gold electrode is always on the left side (dotted line). a) PVDF $9 \mu \mathrm{m}$, positive polarity of the permeable gold electrode, b) PVDF $9 \mu \mathrm{m}$, negative polarity, c) PVDF $25 \mu \mathrm{m}$, positive polarity, d) PVDF $25 \mu \mathrm{m}$, negative polarity.

Additionally to $\mathrm{HF}$, hydrogene $\left(\mathrm{H}_{2}\right)$ and fluorine $\left(\mathrm{F}_{2}\right)$ gas are created during poling of PVDF at the negative and the positive electrode, respectively [28]. A detailed analysis of the gas emission data shows that the $\mathrm{H}_{2}$ and $\mathrm{F}_{2}$ gas emission is a result of the electrochemical creation of $\mathrm{H}^{+}$and $\mathrm{F}^{-}$ions and their transport during poling. The strong formation of $\mathrm{HF}$ during poling [35] which was also found in some independent measurements [36] can be attributed to a zipper-like chain reaction at the polymer chain catalyzed by $\mathrm{F}^{-}$-ions $[37,35]$. The polyene sequences resulting from this reaction can be detected using raman spectroscopy. This is evident from the growth of two raman lines around 1515 $\mathrm{cm}^{-1}$ and $1118 \mathrm{~cm}^{-1}$ with increasing poling strength in Fig. 16.

Poling PVDF in high electrical field the injected homo charges are only sufficient for the initial compensation at the sample boundaries. The resulting additional production of HF during poling possibly provides the ex- cess of $\mathrm{H}^{+}$and $\mathrm{F}^{-}$ions necessary to develop a neutralization of all polarized crystallites in the sample interior.

\section{Summary}

From a series of experimental results revealed within the last 15 years we obtained evidence that the injection of ionic space charge plays the dominant role for the stabilization and in some cases also for the formation of polarization in PVDF. Particularly, charge trapping at the boundaries of the polarized crystallites is the essential mechanism for polarization stabilization. This indicates, that both, homocharges and heterocharges (dipoles) are important in their respective ways for the piezoelectricity and related effects in the polymeric electret PVDF. 


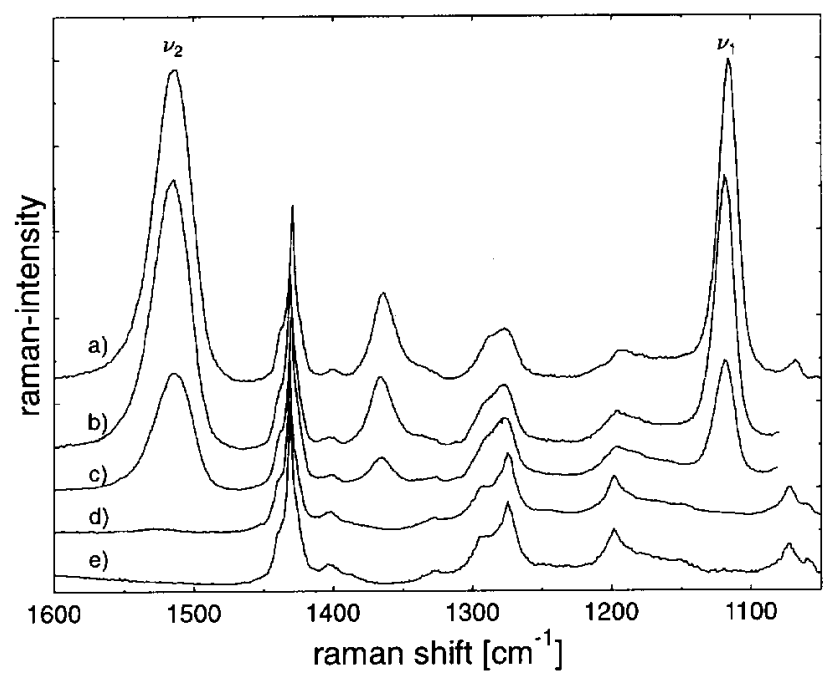

Figure 16. Raman spectra of poled and unpoled $25 \mu \mathrm{m}$ thick PVDF films [37]. Sample a): poled $24 \mathrm{~h}$ with $80 \mathrm{MV} / \mathrm{m}$ at $60^{\circ} \mathrm{C}$; sample b): poled $22 \mathrm{~h}$ with $80 \mathrm{MV} / \mathrm{m}$ at $60^{\circ} \mathrm{C}$; sample c): poled $4 \mathrm{~h}$ with $80 \mathrm{MV} / \mathrm{m}$ at $80^{\circ} \mathrm{C}$; sample d): unpoled: $24 \mathrm{~h}$ at $66^{\circ} \mathrm{C}$ in vacuum; sample e): completely untreated. The spectra are normalized with respect to the line at $1430 \mathrm{~cm}^{-1}$, which can bei attributed to a $\mathrm{CH}_{2}$-deformation vibration.

\section{Acknowledgements}

The results presented in this review originate from a large number of diploma and Phd thesis' by (in alphabetical order) D. Appel, E. Bihler, W. Burger, E. Brazel, B. Dehlen, G. Eberle, D. Günther, M. Haardt, K. Holdik, J. Kringler, B. Küssner, M.-A. Maushart, I. Müller, G. Neumann, H. Schmidt, M. Schmidt, M. Selle and M. Womes. The work was in most parts financially supported by the Deutsche Forschungsgemeinschaft. We also would like to thank our colleagues G.M. Sessler, R. Gerhard-Multhaupt, A.S. DeReggi, J. West and B. Gross for their fruitful and inspiring collaboration.

\section{References}

[1] B. Gross, Experiments on electrets, Phys. Rev. 66, 26 (1944).

[2] B. Gross, L. Ferreira Denard, On permanent charges in solid dielectrics, I. dielectric absorption and temperaure effects in carnauba wax, Phys. Rev. 67, 253 (1945).

[3] B. Gross, Charge Storage in Solid Electrets, Elsevier Publ. Comp., Amsterdam London New York (1964).
[4] B. Gross, Electret Research-Stages in its Development, Proc. 5th Int. IEEE Symp. Electrets, Heidelberg, 9 (1985).

[5] B. Gross, On permanent charges in solid dielectrics, II. surface charges and transient currents inc carnauba wax, J. Chem. Phys. 17, 866.

[6] B. Gross, R.J. de Moraes, Polarization of the electret, J. Chem. Phys. 37, 710 (1962).

[7] K. Holdik, Ladungs- und Polarisationsdynamik in Polymerflmen, $\mathrm{PhD}$ Thesis (in german), University of Stuttgart, 1985.

[8] H. Kawai, The piezoelectricity of poly(vinylidene fluoride), Jpn. J. Appl. Phys. 8, 975 (1969).

[9] G. M. Sessler, D. K. Das-Gupta, A. S. DeReggi, W. Eisenmenger, T. Furukawa, J. A. Giacometti, R. Gerhard-Multhaupt, Piezo- and Pyroelectricity in Electrets, Caused by Charges, Dipoles or both?, IEEE Trans. Electr. Insul. 27, 872 (1992).

[10] A. J. Lovinger, Poly (vinylidene fluoride), In: D. C. Basset(ed.), Developments in Crystalline Polymers, J. Appl. Sci., 1, chapter 5, (1982).

[11] G. M. Sessler (Ed.), Topics in Applied Physics: Electrets, 2nd Ed., Springer, Berlin Heidelberg New York, 1987

[12] G. Eberle, H. Schmidt, W. Eisenmenger, Piezoelectric Polymer Electrets, IEEE Trans. Diel. Electr. Insul. 3, 624 (1996).

[13] D. Naegele, D. Y. Yoon, Orientation of crystalline dipoles in poly(vinylidene fluoride) films under electric field, Appl. Phys. Lett. 33, 132 (1978).

[14] R. G. Kepler, R. A. Anderson, Ferroelectricity in polyvinylidene fluoride, J. Appl. Phys. 49, 1232 (1978).

[15] J. P. Luongo, Far-Infrared Spectra of Piezoelectric Polyvinylidene Fluoride, J. Pol. Sci. 10, part A-2, 1119 (1972).

[16] H. Sussner, K. Dransfeld, Importance of the metalpolymer inerface for the piezoelectricity of polyvinylidene fluoride, J. Polym. Sci. Polym. Phys. 16529 (1978).

[17] R. Gerhard-Multhaupt, Electrets: Dielectrics with quasi-permanent chare or polarization, IEEE Trans. Electr. Ins. 22, 531 (1987).

[18] W. Eisenmenger, M. Haardt, Observation of charge compensated polarization zones in polyvinylidenefluoride $(P V D F)$ by piezoelectric acoustic step-wave response, Solid State Comm. 41, 917 (1982).

[19] K. Holdik, W. Eisenmenger, Charge and polarization dynamics in polymer films, Proc. 5th Int. IEEE Symp. Electrets, Heidelberg, 553-558 (1985). 
[20] A. Becker, M. Stein, B. Jungnickel, Dependece on supermolecular structure and on charge injection conditions of ferroelectric switsching of PVDF and its blends with PMMA, Ferroelectrics, 71, 111 (1995).

[21] G. Eberle, B. Dehlen, W. Eisenmenger, Time development of multiple polarization zones in PVDF, Ultrasonics Symposium Proceedings, 1, 529 (1993).

[22] E. Bihler, G. Neumann, G. Eberle, W. Eisenmenger, Influence of charge injection on the formation of remanent polarization in $P(V D F-\operatorname{TrFE})$ copolymers, IEEE Annual Report Conf. Electr. Insul. Diel. Phenom. 1990, Piscataway, 140 (1990).

[23] M. Womes, E. Bihler, W. Eisenmenger, Dynamics of polarization growth and reversal in PVDF flms, IEEE Trans. Electr. Ins. 24, 461 (1989).

[24] G. Eberle, E. Bihler, W. Eisenmenger, Polarization dynamics of $P(V D F-T r F E)$ copolymers, IEEE Trans. Electr. Ins. 26, 69 (1991).

[25] B. Dehlen, B. Küssner, G. Eberle, W. Eisenmenger, Heat pulse depolarization and short time pyroelectricity of PVDF and copolymer, Proc. 9th Int. IEEE Symp. Electrets, 925-930, Piscataway (1996).

[26] G. Eberle, W. Eisenmenger, Thermal Depolarization of PVDF: Anomaly at $180^{\circ} \mathrm{C}$, IEEE Trans. Electr. Ins. 27, 768 (1992).

[27] B. Dehlen, Räumliche und energetische Verteilung der gebundenen Ladungen in PVDF und in dem Copolymer $P(V D F-\operatorname{Tr} F E), \mathrm{PhD}$ Thesis (in german), University of Stuttgart, 1998.

[28] W. Eisenmenger, B. Dehlen, H. Schmidt, The role of charges for polarization stability in PVDF, to be published, 1998, [27, 37].
[29] B. Küssner, B. Dehlen, G. Eberle, W. Eisenmenger, inding Energies of Trapped Charges in PVDF and $L B P(V D F / \operatorname{TrFE})$, Proc. 8th Int. IEEE Symp. Electrets, Piscataway, 594-599 (1994).

[30] B. Dehlen, Influence of solvents on the polarization distribution in PVDF, IEEE Annual Report Conf. Electr. Insul. Diel. Phenom. 1992, Piscataway, 172-177 (1992).

[31] M. Teda, Electrical conduction and carrier traps in polymeric materials, IEEE Trans. Electr. Insul. 19, 162 (1984).

[32] J. Kringler, Druckabhängigkeit der Leitfähigkeit von Polyvinylidenfluorid (PVDF), Thesis (in german), University of Stuttgart, 1984.

[33] E. Bihler, K. Holdik, W. Eisenmenger, Electric FieldInduced Gas Emission From PVDF Films, IEEE Trans. Electr. Insul. EI-22, 207 (1987).

[34] H. Schmidt, G. Eberle, W. Eisenmenger, Charge detrapping in PVDF observed by field induced gas emission, IEEE Annual Report Conf. Electr. Insul. Diel. Phenom. 1995, Piscataway, 626 (1995).

[35] H. Schmidt, W. Eisenmenger, Internal charge generation in polyvinylidene fluoride during poling oberved by raman spectroscopy, to be published, 1998.

[36] N. Tsutsumi, G. T. Davis, A. S. DeReggi, Protonation of dyes in ferroelectric copolymer of vinylidene fluoride and trifluoroethylene, Polym. Comm. 32, 113 (1991).

[37] H. Schmidt, Ionische Ladungsträger im piezoelektrischen Polymer PVDF, PhD Thesis (in german), University of Stuttgart, 1997.

[38] M. Selle, G. Eberle, B. Gompf, W. Eisenmenger, Field induced gas emission of polymer films, Conf. Electr. Insul. Diel. Phen. (CEIDP) Annual Report (1992) 87-92. 